\title{
Data Augmentation to Improve the diagnosis of Melanoma using Convolutional Neural Networks
}

\author{
YIFAN YANG \\ Division of Biological Sciences, University of California, San Diego, USA Email: bjbzyyf@sina.com
}

\begin{abstract}
Early diagnosis of melanoma can substantially increase patient survival rate. Currently, dermoscopy is the dominant approach for clinical detection, but this method requires interaction with a trained clinical professional resulting in a financial burden which is a major limiting factor for many patients, especially those in remote and rural locations. It has been proposed that deep convolutional neural networks (CNNs) could allow an automated approaches for diagnosis of melanoma. However, there has been limited work regarding the use of CNNs to diagnose melanoma due to a limited amount of labelled training data available, a major limiting factor for the implementation of CNNs. This study utilises data augmentation techniques to improve $\mathrm{CNN}$ performance for diagnosis of melanoma, resulting a $12.4 \%$ increase in validation accuracy despite the collection of no additional training data.
\end{abstract}

\section{CCS Concepts}

- Computing methodologies $\sim$ Machine learning $\sim$ Machine learning approaches $\sim$ Neural networks.

\section{Additional Key Words and Phrases}

Melanoma, Skin cancer, Convolutional Neural Networks (CNNs), Data augmentation

\section{INTRODUCTION}

Melanoma is a typical skin-related cancer, according to the NIH statistics the rate of new cases has been increasing since 1975 and currently results in approximately $75 \%$ of all skin-cancer-related deaths [1]. The 10 year survival rate of patients with melanoma detected at stage IV is $39 \%$, increasing to $93 \%$ for patients diagnosed earlier at Stage I [2]. However, many melanoma

Permission to make digital or hard copies of all or part of this work for personal or classroom use is granted without fee provided that copies are not made or distributed for profit or commercial advantage and that copies bear this notice and the full citation on the first page. Copyrights for components of this work owned by others than ACM must be honored. Abstracting with credit is permitted. To copy otherwise, or republish, to post on servers or to redistribute to lists, requires prior specific permission and/or a fee. Request permissions from Permissions@acm.org.

BIC 2021, January 22-24, 2021, Harbin, China

(C) 2021 Association for Computing Machinery.

ACM ISBN 978-1-4503-9000-2/21/01 ..\$15.00

https://doi.org/10.1145/3448748.3448773 patients miss the opportunity for early diagnosis treatment due to the embarrassment or financial limitations preventing their access to healthcare for early diagnosis.

Historically, melanoma was diagnosed holistically by visual inspection. However, there are some deficiencies in this diagnostic approach. For example, a melanoma less than $5 \mathrm{~mm}$ may not be detected, or some lesions could be falsely classified to benign based on ABCD rule introduced in the 1980s [4]. Dermoscopes can allow examination of melanoma in greater detail and improved diagnostic sensitivity, but this method is highly dependent on the availability of experienced clinicians. Work has been performed to develop automated classification and segmentation of melanoma from dermoscopy images, but training set sizes are limited resulting in poor performance that cannot substitute a clinical examination [5].

In the past decade, deep learning has experienced a huge revolution, deep learning enables the construction of highly generalized models inspired by the human brain's neural networks. The widely used learning architecture is the Convolutional Neural Networks (CNNs) because of the classification, segmentation, and object detection functions [6]. Image detection and classification have been applied showing strong ability of the algorithms promotes a CNN-based projection $[7,8]$.

Machine learning techniques can provide an automated diagnosis of melanoma which could be performed at home using a smartphone appliance, matching the goal to create a quick way for patients to perform an initial check for melanoma. However, modern machine learning techniques require large amounts of training data, while the size of the current training database is limited, transformation is utilized as a data augmentation method as well as can increase the variations of datasets. The aim of this study is to examine multiple transformations to identify data augmentation techniques which improve model performance.

\section{METHODS}

\subsection{Datasets}

The dataset was extracted from Kaggle open platform ${ }^{1}$, containing images either in .jpg or in .jpeg format, which are machine readable file formats and metadata.

${ }^{1}$ https://www.kaggle.com/ghulammujtaba000/melanoma-and-benign-images-only 
2.1.1 Data processing. The original images were stored in RGB mode, where each image has three channels: red, green and blue. Each image is defined in either benign or malignant categories which we attempt to predict with the trained model. For each image, it is defined in either benign or malignant categories. A label was assigned to each image according to the category with a certain numerical value. Here, malignant was assigned to 0 , whereas benign was assigned to 1 .

2.1.2 Data split. The original dataset is split into three sections, denoted as train, test and validation. The testing and validation datasets are independent from the training dataset, the testing and validation sets is used to assess model performance to ensure that the trained model is able to generalize to new data. The partition of the datasets is shown in Table 1: roughly $60 \%$ was allocated to training dataset, $20 \%$ was allocated to testing dataset, and $20 \%$ was allocated to validation dataset.

Table 1. Number of Images of each class in the training, testing, and validation set.

\begin{tabular}{l|l|l|l}
\hline Class / Datasets & Train & Test & Validation \\
\hline Benign & 5341 & 1780 & 1781 \\
\hline Malignant & 5341 & 1780 & 1781 \\
\hline
\end{tabular}

2.1.3 Data normalization and augmentation. All the images were all resized and normalized utilizing the torchvision package in Python. All images were transformed to square 244 by 244 pixels. Furthermore, a range of image transformations were applied to the images. These data augmentation techniques artificially increase the diversity of the existing images by manipulating the features. Multiple transformations were performed to test the potential effects generated on the model performance.

\subsection{Algorithms}

The code was implemented as illustrated in the following steps:

(1) Import all needed packages-torch, torchvision, sklearn

(2) Transformation List ${ }^{2}$

(3) Data Loader

(a)Train Loader with batch_size $=8$

(b) Test Loader with batch_size $=50$

(c) Validation Loader with batch_size $=50$

(4) Visualization Function
(5) Train Images Iterator

(6) Custom CNNs Architecture ${ }^{43}$

(7) Loop over dataset 20 times $($ epoch $=20)$

(a) Training Loss

(b) Training F1_score

(c) Training Accuracy_score

(d) Validation Loss

(e) Validation F1_score

(f) Validation Accuracy_score

\subsection{Convolutional Neural Networks Architecture}

Classifying the melanoma images without an established criteria is difficult, so incorporating CNNs as a tool in the diagnosis task would be beneficial for detecting or predicting potential lesions.

There are three components that can define a basic convolutional neural network. The images loaded can be regarded as an array of pixel values. In a convolutional layer, a self-defined weight matrix will extract the certain features from the images, which run across the image so that all the pixels are covered at least once, to give a convolved output. Another component is the pooling layer. The sole purpose is to reduce the spatial size of images by compressing features relevant to prediction of the desired outcome. Pooling is done on each depth of dimension independently. Finally, after multiple layers, the output layer is used to generate an output equal to the number of classes needed. The loss function like cross-entropy is used to compute the error in prediction [9]. Using the loss function a gradient descent algorithm is applied for iterative adjustment of the model weights, which inform the convolution process, in order to minimise the calculated loss resulting in increased model performance.

\subsection{Custom CNN Architecture}

The custom architecture implied contains 7 layers, 2 of which are convolutional layers, 2 pooling layers and 2 linear layers, and a final output layer with two neurons representing a benign or malignant diagnosis.

The first convolutional layer has three input channels reflecting the RGB input images, generates six output channels by convolution with a $(5 \times 5)$ kernel, followed by a ReLU activation function, the images are passed to the first

${ }^{3}$ More details will be introduced more in 2.4 
max pooling layer, with square window of size 2 and stride value of 2. Following pooling is a second convolutional layer, six input channels are loaded from the number of output channels from the first convolutional layer. After this second convolution, sixteen output channels are produced. A kernel size of five was chosen, to be the same as the first convolutional layer. Again called by a ReLU activation function, the processed images are loaded to the second max pooling layer. All convolutional layers utilized a default stride and padding of one and zero respectively [10].

After the feature learning process, the next operation is to flatten all the output channels represented as square matrices into a one-dimensional vector. Constructing two linear layers to have a linear transformation of 2D data, finally softmax the vector before passing all the results to the output layer.

\subsection{Model Performance Metrics}

If the model correctly categorizes melanoma as benign, the prediction is true positive (TP); if the model correctly predicts a benign melanoma the prediction is false positive (FP). In a similar way, a false negative (FN) is the model predicts the melanoma as malignant but benign actually, and a true negative (TN) is the model correctly classifies malignant melanoma. These four primary parameters, as illustrated in Table 2, are the foundations of other measures of prediction performance.

Table 2. Primary Parameters

\begin{tabular}{l|l|l}
\hline $\begin{array}{l}\text { Model Prediction / True } \\
\text { situation }\end{array}$ & Benign & Malignant \\
\hline Benign & True Positive & False Positive \\
\hline Malignant & False Negative & True Negative \\
\hline
\end{tabular}

The computational definition of precision is shown in equation 1 , which measures the ratio of the number of images correctly classified as benign to the total number of predicted results as benign.

$$
\begin{gathered}
T P \\
\text { precision }= \\
T P+F P
\end{gathered}
$$

Recall can be calculated as illustrated in equation 2 , which represents the ratio of the number of images correctly classified as benign to the total number of actual results as benign.

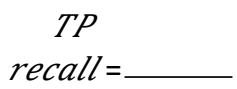

$$
T P+F N
$$

Accuracy score and F1 score are more complex than precision and recall and provides a more comprehensive measurement of model performance. The formula are displayed in equation 3 and equation 4 . Accuracy score takes account the proportion of all correct predictions, while F1 score is the harmonic mean of precision and recall since sometime it is necessary to create a balance between the false positive and false negative.

$$
\begin{gathered}
T P+T N \\
\text { Accuracy_score } \\
=\frac{T P+T N+F P+F N}{\text { precision } \cdot \text { recall }} \\
F 1=2 \cdot \frac{\text { precision }+ \text { recall }}{}
\end{gathered}
$$

\section{RESULTS}

No transformation is used as a referencing baseline, adding data transformations can result in increases or decreases in model performance. A random horizontal flip with a probability of 0.5 , random grayscale, random crop improve the model performance dramatically. However, random horizontal flip with a probability of 0.8 , and random vertical flip with a probability of 0.2 decreases the model performance (Table 5).

\subsection{CNNs differentiate between malignant and benign melanoma with a F1 score of $84 \%$}

Applying total 9 distinct transformations, which the functions are explained below:

I Flip and Rotation

This group of transformations change the direction of melanoma images.

(a) Random Horizontal Flip

Flip the image horizontally with probability $\mathrm{p}$

(b) Random Vertical Flip

Flip the image horizontally with probability $\mathrm{p}$

(c) Random Rotation

Rotate at a random angle according to the degrees

II Crop 
This group of transformations change the size of melanoma images.

(a) Random Crop

Random cropping according to the given size

The parameter padding (int) is to set how many pixels are padded.

(b) Center Crop

Cropping from the center according to the given size

III Modify images

This group of transformations change the feature of melanoma images.

(a) Color Jitter

Randomly change the brightness, contrast and saturation of an image

(b) Random Grayscale

Converts images to grayscale with probability $\mathrm{p}$

(c) Random Affine

Affine transformation

(d) Random Perspective

Performs a random perspective transformation of the given image with a given probability

The loss function is calculated with cross-entropy using pytorch, while accuracy score and F1 score through the corresponding function in Sci-Kit Learn package. Table 3 and Table 4 recorded all data from tensorboard visualization.

\subsection{Horizontal flip transformation increases CNNs validation $F 1$ score by $8.79 \%$ compared to no transformation}

In Table 5 and Table 6, no transformation was set as a reference frame, the manifestations of all other transformations can be easily seen. Calculated from the following formula, all processed data are recorded in Table 5 and Table $6^{4}$.

$$
\begin{gathered}
\text { trans }- \text { no_trans } \\
\text { percentage_change }= \\
\text { no_trans }
\end{gathered}
$$

Table 3. Training Results

\begin{tabular}{l|l|l|l}
\hline Method / Function & Ac Score & F1 Score & Loss \\
\hline No trans & 6.05 & 0.76 & 0.58
\end{tabular}

\footnotetext{
${ }^{4}$ All data have been rounded to two decimal places.
}

\begin{tabular}{l|l|l|l} 
Hori flip $(0.2)$ & 6.74 & 0.83 & 0.47 \\
Hori flip(0.5) & 6.82 & 0.84 & 0.46 \\
Hori flip(0.8) & 6.22 & 0.77 & 0.60 \\
Vert flip(0.2) & 6.21 & 0.77 & 0.60 \\
Vert flip $(0.5)$ & 6.60 & 0.81 & 0.48 \\
Vert flip $(0.8)$ & 6.56 & 0.80 & 0.49 \\
Rad rotation $\left( \pm 90^{\circ} \mathrm{C}\right)$ & 6.59 & 0.81 & 0.48 \\
Rad grayscale & 6.82 & 0.83 & 0.45 \\
Color jitter & 6.53 & 0.81 & 0.48 \\
Rad affine & 6.59 & 0.81 & 0.48 \\
Rad perspective & 6.48 & 0.80 & 0.49 \\
Rad crop & 6.79 & 0.83 & 0.46 \\
Rad crop(pad=4) & 6.65 & 0.82 & 0.48 \\
Rad crop(pad=8) & 6.53 & 0.79 & 0.48 \\
Ctr crop(pad=4) & 6.68 & 0.82 & 0.47 \\
Ctr crop(pad=8) & 6.76 & 0.82 & 0.46 \\
\hline
\end{tabular}

Table 4. Validation Results

\begin{tabular}{l|l|l|l}
\hline Method / Function & Ac Score & F1 Score & Loss \\
\hline No trans & 37.38 & 0.77 & 0.59 \\
Hori flip(0.2) & 41.28 & 0.82 & 0.48 \\
Hori flip(0.5) & 42.01 & 0.84 & 0.47 \\
Hori flip(0.8) & 38.16 & 0.78 & 0.61 \\
Vert flip(0.2) & 38.98 & 0.79 & 0.60 \\
Vert flip(0.5) & 40.73 & 0.82 & 0.49 \\
Vert flip(0.8) & 39.14 & 0.79 & 0.52 \\
Rad rotation( $\left.\pm 90^{\circ} \mathrm{C}\right)$ & 41.61 & 0.83 & 0.47 \\
Rad grayscale & 41.63 & 0.83 & 0.47 \\
Color jitter & 39.61 & 0.80 & 0.50 \\
Rad affine & 41.00 & 0.83 & 0.49 \\
Rad perspective & 40.28 & 0.81 & 0.50 \\
Rad crop & 41.21 & 0.82 & 0.48 \\
Rad crop(pad=4) & 40.71 & 0.82 & 0.50 \\
Rad crop(pad=8) & 40.25 & 0.82 & 0.49 \\
Ctr crop(pad=4) & 40.70 & 0.82 & 0.49 \\
Ctr crop(pad=8) & 41.20 & 0.82 & 0.48 \\
\hline
\end{tabular}


The best transformations are random horizontal flip with a probability of 0.5 , random grayscale, and random crop, which increase the model performances by $20.70 \%, 20.11 \%, 18.87 \%$, respectively. The worst transformations are random horizontal flip with a probability of 0.8 , and random vertical flip with a probability of 0.2 , which decrease the model performances by $3.02 \%, 1.58 \%$, respectively.

\section{Table 5. Training percentage_change compared to NO trans}

\begin{tabular}{l|l|l|l}
\hline Method / Function & Ac Score & F1 Score & Loss \\
\hline Hori flip(0.2) & $+11.56 \%$ & $+9.45 \%$ & $-20.07 \%$ \\
Hori flip(0.5) & $+12.87 \%$ & $+10.72 \%$ & $-21.53 \%$ \\
Hori flip(0.8) & $+2.91 \%$ & $+2.29 \%$ & $+3.66 \%$ \\
Vert flip(0.2) & $+2.76 \%$ & $+1.73 \%$ & $+3.36 \%$ \\
Vert flip(0.5) & $+9.16 \%$ & $+6.74 \%$ & $-17.96 \%$ \\
Vert flip(0.8) & $+8.57 \%$ & $+5.92 \%$ & $-16.41 \%$ \\
Rad rotation( $\left.\pm 90^{\circ} \mathrm{C}\right)$ & $+8.95 \%$ & $+6.71 \%$ & $-16.96 \%$ \\
Rad grayscale & $+12.79 \%$ & $+10.49 \%$ & $-22.25 \%$ \\
Color jitter & $+7.99 \%$ & $+6.62 \%$ & $-17.22 \%$ \\
Rad affine & $+9.00 \%$ & $+6.74 \%$ & $-16.93 \%$ \\
Rad perspective & $+7.21 \%$ & $+5.32 \%$ & $-15.26 \%$ \\
Rad crop & $+12.24 \%$ & $+9.93 \%$ & $-21.51 \%$ \\
Rad crop(pad=4) & $+10.06 \%$ & $+8.34 \%$ & $-18.33 \%$ \\
Rad crop(pad=8) & $+8.09 \%$ & $+5.20 \%$ & $-17.25 \%$ \\
Ctr crop(pad=4) & $+10.52 \%$ & $+8.59 \%$ & $-19.78 \%$ \\
Ctr crop(pad=8) & $+11.75 \%$ & $+8.69 \%$ & $-20.86 \%$ \\
\hline
\end{tabular}

Table 6. Validation percentage_change compared to NO trans

\begin{tabular}{l|l|l|l}
\hline Method / Function & Ac Score & F1 Score & Loss \\
\hline Hori flip(0.2) & $+10.43 \%$ & $+6.30 \%$ & $-18.38 \%$ \\
Hori flip(0.5) & $+12.39 \%$ & $+8.79 \%$ & $-20.70 \%$ \\
Hori flip(0.8) & $+2.09 \%$ & $+0.92 \%$ & $+3.02 \%$ \\
Vert flip(0.2) & $+4.28 \%$ & $+2.23 \%$ & $+1.58 \%$ \\
Vert flip(0.5) & $+8.96 \%$ & $+6.25 \%$ & $-17.45 \%$ \\
Vert flip(0.8) & $+4.71 \%$ & $+2.70 \%$ & $-11.30 \%$ \\
Rad rotation( $\left.\pm 90^{\circ} \mathrm{C}\right)$ & $+11.32 \%$ & $+8.36 \%$ & $-19.57 \%$ \\
Rad grayscale & $+11.37 \%$ & $+7.99 \%$ & $-20.11 \%$ \\
Color jitter & $+5.97 \%$ & $+4.12 \%$ & $-15.25 \%$ \\
Rad affine & $+9.68 \%$ & $+7.61 \%$ & $-17.52 \%$ \\
Rad perspective & $+7.76 \%$ & $+5.00 \%$ & $-16.03 \%$ \\
Rad crop & $+10.25 \%$ & $+6.88 \%$ & $-18.87 \%$
\end{tabular}




\begin{tabular}{l|l|l|l}
$\operatorname{Rad} \operatorname{crop}(\mathrm{pad}=4)$ & $+8.91 \%$ & $+6.57 \%$ & $-16.08 \%$ \\
$\operatorname{Rad} \operatorname{crop}(\mathrm{pad}=8)$ & $+7.68 \%$ & $+5.84 \%$ & $-16.50 \%$ \\
$\mathrm{Ctr} \operatorname{crop}(\mathrm{pad}=4)$ & $+8.88 \%$ & $+6.60 \%$ & $-17.58 \%$ \\
$\mathrm{Ctr} \operatorname{crop}(\mathrm{pad}=8)$ & $+10.22 \%$ & $+6.70 \%$ & $-18.24 \%$ \\
\hline
\end{tabular}

Based on the calculated definitions above, the wanted result is the loss value as low as possible, and the F1 score and accuracy score as high as possible. Among all these transformations, the best three (labeled in blue) and worst three (labeled in red) are chosen.
Figure 1 gathered the best and worst results from the tensorboard visulization. Top half: Training sets. Bottom half: validation sets. Accuracy score, F1 score, loss value from left to right. $\mathrm{X}$-axis and $\mathrm{Y}$-axis are labeled in the graph. Each line is displayed in different color with a side annotation.
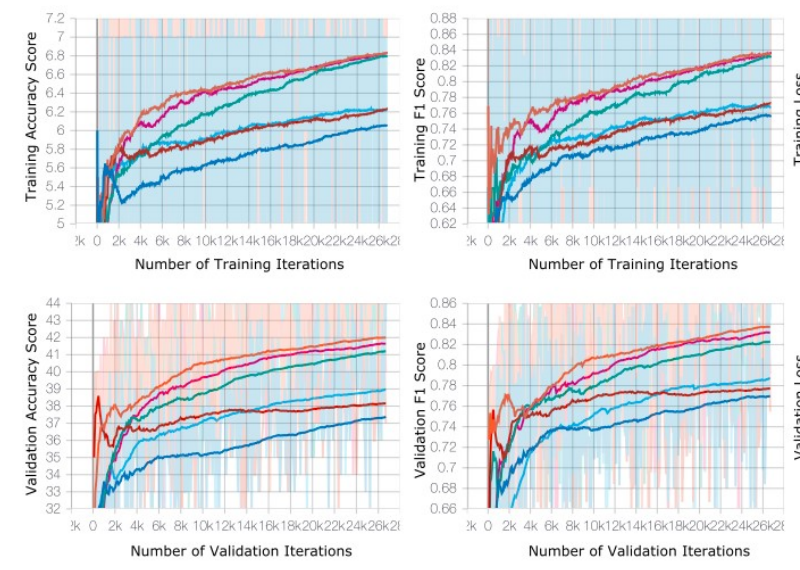

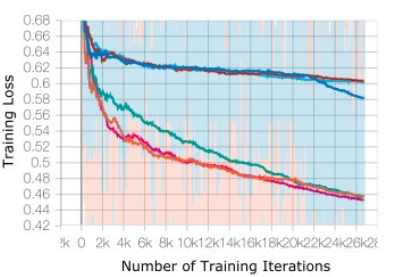

Nandom Horizontal Flip $(\mathrm{p}=0.5)$ - No Transformation - Random Horizontal Flip $(p=0.8)$ Random Vertical Flip $(p=0.2)$ - Random Grayscale In Random Crop

Fig. 1. Custom CNNs Architecture Results

\subsection{Combining top performing transformation results combining transformations did not result in increased performance?}

Further exploration of the best three transformations were done to test if multiple transformations applied together would result in further improvements in model performance. Four combinations of 2 and 3 simultaneous transformations were tested. In section 3.2, the best transformation improved the model performances by $20.70 \%$, while the best transformation among the four new combinations improved the model performances by $20.34 \%$. Therefore, combining the best transformation did not help to improve the model performances significantly.

\section{Table 7. Overlay Training Results}

\begin{tabular}{l|l|l|l}
\hline Method / Function & Ac Score & F1 Score & Loss \\
\hline Hori+grayscale & 6.34 & 0.79 & 0.49 \\
Hori+crop & 6.73 & 0.82 & 0.47 \\
grayscale+crop & 6.81 & 0.83 & 0.46 \\
Hori+gray+crop & 6.65 & 0.81 & 0.47 \\
\hline
\end{tabular}

Table 8. Overlay Validation Results

\begin{tabular}{l|l|l|l}
\hline Method / Function & Ac Score & F1 Score & Loss \\
\hline Hori+grayscale & 38.80 & 0.80 & 0.50 \\
Hori+crop & 41.50 & 0.83 & 0.47 \\
grayscale+crop & 41.51 & 0.81 & 0.48 \\
Hori+gray+crop & 41.34 & 0.83 & 0.48 \\
\hline
\end{tabular}

Table 9. Overlay Training percentage_change compared to NO trans

\begin{tabular}{l|l|l|l}
\hline Method / Function & Ac Score & F1 Score & Loss \\
\hline Hori+grayscale & $+4.79 \%$ & $+3.95 \%$ & $-15.52 \%$ \\
Hori+crop & $+11.24 \%$ & $+7.89 \%$ & $-18.97 \%$ \\
grayscale+crop & $+12.56 \%$ & $+9.21 \%$ & $-20.69 \%$ \\
Hori+gray+crop & $+9.92 \%$ & $+6.58 \%$ & $-18.97 \%$ \\
\hline
\end{tabular}


Table 10. Overlay Validation percentage_change compared to NO trans

\begin{tabular}{l|l|l|l}
\hline Method / Function & Ac Score & F1 Score & Loss \\
\hline Hori+grayscale & $+3.80 \%$ & $+3.90 \%$ & $-15.25 \%$ \\
Hori+crop & $+11.02 \%$ & $+7.79 \%$ & $-20.34 \%$
\end{tabular}
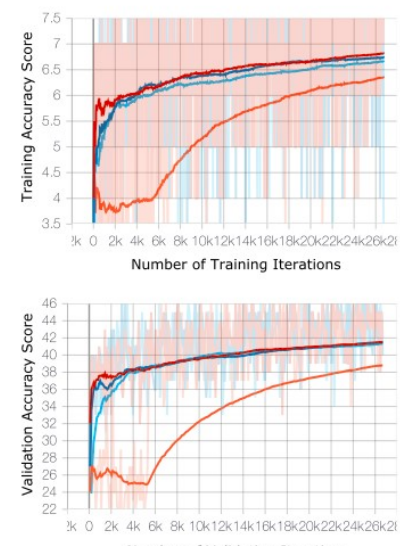

Number of Validation Iterations
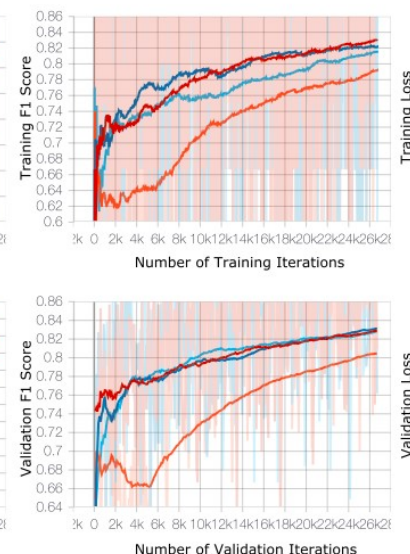

Number of Validation Iterations

\begin{tabular}{l|l|l|l} 
grayscale+crop & $+11.05 \%$ & $+5.19 \%$ & $-18.64 \%$ \\
Hori+gray+crop & $+10.59 \%$ & $+7.79 \%$ & $-15.64 \%$ \\
\hline
\end{tabular}

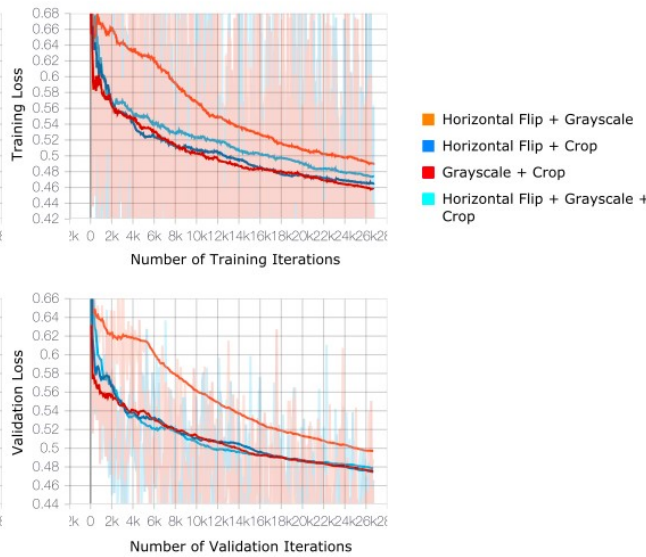

Fig. 2. Overlay Custom CNNs Architecture Results

\section{CONCLUSION AND DISCUSSION}

A major limitations for the development of machine learning techniques for the diagnosis of melanoma is the limiting availability of training data which results in models with poor performance that do not generalize. In this work I have shown that data augmentation techniques improve model performance in the context of training CNNs to differentiate between malignant and benign melanoma. Random horizontal flip, random grayscale and random crop, increase the model performances significantly, while random horizontal flip with a probability of $80 \%$, and random vertical flip with a probability of $20 \%$ downregulate the model performances.

Although I show that data augmentation techniques result in a significant increase in model performance, the accuracy achieved is too low for deployment of the these models in a clinical setting $(41.51 \%)$. The purpose of this work is to assess the role of data augmentation in improving model performance which will contribute to the deployment of models in a clinical setting in the future.

The constructed CNNs (Section 2.3) utilized in this study is limited compared to larger production CNNs such as ResNet18, AlexNet, and VGG16 [11]. For future work, a comparative analysis will be conducted using the same dataset to see the difference.

\section{REFERENCES}

[1] Esteva, A., Kuprel, B., Novoa, R. A., Ko, J., Swetter, S. M., Blau, H. M., \& Thrun, S. (2017). Dermatologist-level classification of skin cancer with deep neural networks $\quad$ Nature, 542(7639), 115-118. https://doi.org/10.1038/nature21056 2. Balch, C. M., Gershenwald, J. E., Soong, S. J., Thompson, J. F., Atkins, M. B., Byrd, D. R., Buzaid, A. C., Cochran, A. J., Coit, D. G., Ding, S., Eggermont, A. M., Flaherty, K. T., Gimotty, P. A., Kirkwood, J. M., McMasters, K. M., Mihm, M. C., Jr, Morton, D. L., Ross, M. I., Sober, A. J., \& Sondak, V. K. (2009). Final version of 2009 AJCC melanoma staging and classification. Journal of clinical oncology : official journal of the American Society of Clinical Oncology, 27(36),6199-6206. https://doi.org/10.1200/JCO.2009.23.4799

[2] Tchernev G. (2017). Novel Surgical Approach in Cutaneous Melanoma Patients: "Daring Ideas Are Like ChessmenMoved Forward. They May Be Beaten, But they May Start a Winning Game!?". Open access Macedonian journal of medical sciences, 5(6), 810-812. https://doi.org/10.3889/oamjms.2017.199

[3] Argenziano, G., \& Soyer, H. P. (2001). Dermoscopy of pigmented skin lesions-a valuable tool for early diagnosis ofmelanoma. The Lancet. Oncology, 2(7), 443-449. https://doi.org/10.1016/s1470-2045(00)00422-8

[4] Codella, N. C. F., Gutman, D., Celebi, M. E., Helba, B., Marchetti, M. A., Dusza, S. W., Kalloo, A., Liopyris, K., Mishra,N., Kittler, H., \& Halpern, A. (2018). Skin lesion analysis toward melanoma detection: A challenge at the 2017 International symposium on biomedical imaging (ISBI), hosted by the international skin imaging collaboration (ISIC). 2018 IEEE 15th International Symposium on Biomedical Imaging (ISBI 2018). https://doi.org/10.1109/isbi.2018.8363547

[5] Pan, D., Zeng, A., Jia, L., Huang, Y., Frizzell, T., \& Song, X. (2020). Early Detection of Alzheimer's Disease Using Magnetic Resonance Imaging: A Novel Approach Combining Convolutional Neural Networks and Ensemble $\begin{array}{lll}\text { Learning. Frontiers in neuroscience, 14, } 259 . & \end{array}$ https://doi.org/10.3389/fnins.2020.00259

[6] Papandrianos, N., Papageorgiou, E., Anagnostis, A., \& Papageorgiou, K. (2020). Bone metastasis classification usingwhole body images from prostate cancer patients based on convolutional neural networks application. PloS one, 15(8), e0237213. https://doi.org/10.1371/journal.pone.0237213

[7] Gulshan, V., Peng, L., Coram, M., Stumpe, M. C., Wu, D., Narayanaswamy, A., 
Venugopalan, S., Widner, K., Madams,T., Cuadros, J., Kim, R., Raman, R., Nelson, P. C., Mega, J. L., \& Webster, D. R. (2016). Development and

Validation of a Deep Learning Algorithm for Detection of Diabetic Retinopathy in Retinal Fundus Photographs. JAMA, 316(22), 2402. https://doi.org/10.1001/jama.2016.17216

[8] Architecture of Convolutional Neural Networks (CNNs) demystified. Retrieved November 1 ,

2020

fromhttps://www.analyticsvidhya.com/blog/2017/06/architecture-of-

convolutional-neural-networks-simplifieddemystified/

[9] https://pytorch.org/docs/stable/nn.html

[10] Difference between AlexNet, VGGNet, ResNet, and Inception | by . Retrieved November 1, 2020, fromhttps://towardsdatascience.com/the-w3h-of-alexnetvggnet-resnet-and-inception-7baaaecccc 96 